\title{
Deconfining the rotational Goldstone mode: The superconducting quantum liquid crystal in $(2+1)$ dimensions
}

\author{
Aron J. Beekman, ${ }^{1}$ Kai Wu, ${ }^{2,}{ }^{*}$ Vladimir Cvetkovic, ${ }^{3}$ and Jan Zaanen ${ }^{2}$ \\ ${ }^{1}$ RIKEN Center for Emergent Matter Science (CEMS), Wako 351-0198, Japan \\ ${ }^{2}$ Institute-Lorentz for Theoretical Physics, Leiden University, P. O. Box 9506, NL-2300 RA Leiden, The Netherlands \\ ${ }^{3}$ National High Magnetic Field Laboratory and Department of Physics, Florida State University, Tallahassee, Florida 32306, USA
}

(Received 3 February 2013; published 31 July 2013)

\begin{abstract}
The Goldstone theorem states that there should be a massless mode for each spontaneously broken symmetry generator. There is no such rotational mode in crystals; however, superconducting quantum liquid crystals should carry rotational Goldstone modes. By generalization of thermal two-dimensional (2D) defect mediated melting theory into a $(2+1) \mathrm{D}$ quantum duality, the emergence of the rotational mode at the quantum phase transition from the solid to the $p$-atic liquid crystal arises as a deconfinement phenomenon, with the unusual property that the stiffness of the rotational mode originates entirely in the dual dislocation condensate.
\end{abstract}

DOI: 10.1103/PhysRevB.88.024121

PACS number(s): 64.70.Tg, 11.30.Qc, 61.30.Gd, 74.25.N-

\section{INTRODUCTION}

Crystals break both translations and rotations but their Nambu-Goldstone modes (phonons) are translational only. Where are the modes expected from the Goldstone theorem ${ }^{1-3}$ associated with the rotational symmetry breaking? ${ }^{4-6}$ Their absence is crucial to engineering since the presence of massless "rotational phonons" would imply an elastic response towards torque stresses, corrupting crankshafts and so forth. However, there is matter that just breaks space rotations leaving translations intact. Such liquid crystals $s^{5,7-9}$ do exhibit an elastic response towards torque stress. In classical nematics this issue is muddled by the peculiarity that the rotational Goldstone modes are overdamped due to the decay in hydrodynamical rotational flows. ${ }^{8,10}$ In a recent development evidence for the existence of zero-temperature quantum liquid crystals has been accumulating. ${ }^{11,12}$ A particular variety appears to be present in cuprates $^{13-16}$ and iron-arsenides ${ }^{17,18}$ with ground states that are also superconducting. Given that hydrodynamical vorticity is gapped in superconductors, this is a natural theater in which to look for propagating rotational Goldstones.

The conventional theory of liquid crystals departs from a kinetic gas theory perspective, ${ }^{7,8}$ a view which is also popular in the quantum realms. ${ }^{12,19}$ However, a dense liquid crystal might be closer to a system which is locally still like a solid, turning at long distances into a fluid because dislocations (translational topological excitations) have proliferated. This notion was introduced by the famous Kosterlitz-Thouless-Nelson-HalperinYoung theory of the thermal melting of a two-dimensional (2D) floating crystal into the hexatic liquid crystal. ${ }^{20-22}$ More recently, a powerful field-theoretical weak-strong duality ${ }^{23-25}$ was mobilized to address the zero-temperature quantum melting of a bosonic solid into a quantum " $p$-atic superconductor" phase $^{26-29}$ (or " $p$-atic superfluid" for crystals of uncharged particles), where we follow Lubenksy's classification of states with broken orientational but full translational symmetry, e.g., "6-atic" is the hexatic phase of melting a triangular lattice and "2-atic" is the unixial nematic. ${ }^{9}$ This follows closely the pattern of the superfluid-superconductor Abelian-Higgs duality in $(2+1) D .^{26,30}$ The rigidity of the crystal phase is captured by gauge fields (here called stress photons) representing the capacity of the medium to propagate shear forces. These are sourced by the dislocations, and quantum melting into the $p$-atic superconductor corresponds to a proliferation and Bose condensation of dislocations into an effectively relativistic "stress superconductor" explained below. These $(2+1) \mathrm{D}$ systems are reminiscent of the $(3+0) \mathrm{D}$ Abrikosov flux-line lattices; ${ }^{31,32}$ however, in the latter static case the issue of the rotational Goldstone modes was not addressed, and we shall see why.

This theory can be regarded as universal for the longwavelength regime in the adiabatic continuity sense. Here we want to present the counterintuitive but elegant resolution it offers for the phonon conundrum of the first paragraph. We will demonstrate that the "crankshaft rigidity" of the solid is explained as a confinement of torque stress, in the same guise as color forces are confined in the infrared of a compact gauge theory. At the quantum phase transition to the superconductor $p$-atic, the shear forces acquire a Higgs mass $\Omega$ signaling the disappearance of the phonons due to the restoration of the translations. The associated length is the "shear penetration depth," having the same status as the London penetration depth, here specifying the length over which shear forces decay in the quantum $p$-atic. At the same time, the dislocation condensate deconfines the torque stress, indicating the appearance of a massless rotational Goldstone mode, going hand-in-hand with the deconfinement of the sources of torque stress: disclinations (rotational topological defects).

A quite nontrivial prediction follows from the duality: the quantities specifying the collective excitations of the superconductor coincide with those describing the crystal elasticity [Eq. (1)]; the shear Higgs mass is the only additional ingredient. The velocity of the rotational Goldstone is set by the phonon velocity but the rotational (nematic) modulus diverges with $\Omega^{-2}$ approaching the quantum phase transition back to the solid; see Eq. (21). The mechanism explaining this single scale is remarkable: it is rooted in a new form of "Higgsing." The rotational stiffness in the quantum $p$-atic is actually carried by the dislocation condensate - as opposed to as a remnant of the solid-and it knows about the rotational symmetry breaking only because the direction of the Burgers vectors of the 
dislocation condensate are fixed by the original crystal lattice. The "longitudinal Goldstones" (gauged phase modes) of this special condensate rearrange in such a way that, besides being "eaten" by the stress photon, they also form the massless rotational Goldstone mode. Let us use the remainder to explain how this works.

\section{DUAL ELASTICITY}

For simplicity, we depart from a minimal theory of quantum elasticity, describing an isotropic medium in $(2+1) \mathrm{D}$. One combines the gradients of the displacement field $u^{a}(x)$ in symmetric strain tensors $w_{a b}=\frac{1}{2}\left(\partial_{a} u^{b}+\partial_{b} u^{a}\right)$. The Lagrangian describing the static elasticity as well as the acoustic phonons includes a kinetic term $(\tau=i t$ is imaginary time), ${ }^{26}$

$$
\mathcal{L}=\frac{\rho}{2}\left(\partial_{\tau} u^{a}\right)^{2}+\frac{\kappa-\mu}{2}\left(w_{a a}\right)^{2}+\frac{2 \mu}{2}\left(w_{a b}\right)^{2},
$$

in terms of the compression modulus $\kappa$, the shear modulus $\mu$, and the mass density $\rho$, such that the longitudinal and transverse phonons propagate with velocities $c_{\mathrm{L}}^{2}=(\kappa+\mu) / \rho$ and $c_{\mathrm{T}}^{2}=\mu / \rho$, respectively. To establish contact with gauge field theory one focuses on the capacity of the medium to propagate forces, and this is accomplished by strainstress duality. By Legendre transformation one defines the stress tensor $\sigma_{\mu}^{a}=\frac{\delta \mathcal{L}}{\delta\left(\partial_{\mu} u^{a}\right)}$, where $\partial_{\mu} \equiv\left(\frac{1}{c_{\mathrm{T}}} \partial_{\tau}, \partial_{m}\right)$. The dual stress field satisfies the conservation law and Ehrenfest constraint,

$$
\begin{gathered}
\partial_{\mu} \sigma_{\mu}^{a}=0, \\
\epsilon_{a m} \sigma_{m}^{a}=0 .
\end{gathered}
$$

The Ehrenfest constraint Eq. (3) eliminates spontaneous torque, in dual correspondence with the absence of antisymmetric "rotational strains" $\omega=\frac{1}{2}\left(\partial_{x} u^{y}-\partial_{y} u^{x}\right)$ in the leading gradient terms defining the action Eq. (1). Although less familiar in this context, one can introduce gauge fields in the same way as in Abelian-Higgs duality by exploiting the fact that the conservation of stress Eq. (2) can be imposed in $(2+1) \mathrm{D}$ by parameterizing the stress tensor in terms of "flavored" (spatial labels a) $U(1)$ gauge fields $b_{\lambda}^{a}$ as $^{23}$

$$
\sigma_{\mu}^{a}(x)=\epsilon_{\mu \nu \lambda} \partial_{\nu} b_{\lambda}^{a}(x) .
$$

In the case of Abelian-Higgs (or $X Y$ ) duality the unique internal sources for the dual photons are the vortices, with the implication that the $(2+1) \mathrm{D}$ superfluid is mapped onto the electromagnetic Coulomb phase. Similarly, here one finds that the stress photons are sourced by dislocation currents: $\mathcal{L}_{\text {source }}=b_{\mu}^{a} J_{\mu}^{a}$, where $J_{\mu}^{a}=\epsilon_{\mu \nu \lambda} \partial_{\nu} \partial_{\lambda} u_{\text {sing }}^{a}$ and $u_{\text {sing }}^{a}$ is the singular part of the displacement field. ${ }^{23,25}$ Compared to the vortices, this structure is richer since the dislocations carry Burgers vectors as topological invariants, labeled by $a$, encoding that dislocations only restore translations in the direction of their Burgers vectors. The bottom line is that phonons can actually be viewed as the duals of stress photons, describing the exchange of elastic forces between the translational topological defects.

\section{TORQUE STRESS GAUGE FIELD}

The breaking of rotational symmetry is completely implicit in the above derivation. Its influence is embodied by the disclination, the topological defect uniquely associated with the restoration of the isotropy of space. Our main discovery is a generalization of stress gauge field theory, rendering the role of rotational symmetry explicit in terms of a torque stress gauge field. For this we have to treat the Ehrenfest condition Eq. (3) as a dynamical constraint. This imposes that only the symmetric part $\bar{\sigma}_{m}^{a}=\bar{\sigma}_{a}^{m}$ of the full stress tensor $\sigma_{m}^{a}=\bar{\sigma}_{m}^{a}+\breve{\sigma}_{m}^{a}$ is present, while its antisymmetric part $\breve{\sigma}_{m}^{a}=-\breve{\sigma}_{a}^{m}$ vanishes. Our trick is to use the antisymmetric strain $\omega$ as an independent dynamical field by implementing the relation $\omega=\frac{1}{2} \epsilon_{i j} \partial_{j} u^{j}$ as a constraint. We conveniently choose $\epsilon_{m a} \breve{\sigma}_{m}^{a}$ as the Lagrange multiplier and add the term $\epsilon_{m a} \breve{\sigma}_{m}^{a}\left(\omega-\frac{1}{2} \epsilon_{i j} \partial_{i} u^{j}\right)$ to the Lagrangian Eq. (1), dualizing into

$$
\mathcal{L}_{\text {constraint }}=\sigma_{\mu}^{a} \partial_{\mu} u^{a}+\epsilon_{m a} \sigma_{m}^{a} \omega .
$$

As usual the conservation of stress Eq. (2) is imposed by $u^{a}$ as a Lagrange multiplier, ${ }^{26,29}$ but now also the Ehrenfest constraint Eq. (3) is incorporated by integrating out the smooth part of $\omega$. Now comes the crucial step: substitute Eq. (4) into Eq. (3) to obtain the conserved torque stress $\tau_{\mu}$,

$$
\begin{gathered}
\tau_{\mu} \equiv \epsilon_{b a} \epsilon_{b \mu \lambda} b_{\lambda}^{a}, \\
\partial_{\mu} \tau_{\mu}=0 .
\end{gathered}
$$

The conservation of torque Eq. (7) can be imposed by parameterizing it in terms of a single vector $U(1)$ torque stress gauge field $h_{\mu}$ associated with the $O(2)$ rotational symmetry of 2D space,

$$
\tau_{\mu}=\epsilon_{\mu \nu \lambda} \partial_{\nu} h_{\lambda}
$$

The important and notable feature of the torque stress and associated gauge field is that it singles out the rotational degrees of freedom. Earlier works ${ }^{26,29,31}$ address the rotational sector only implicitly via the shear stress.

Substituting the dual gauge fields Eqs. (4) and (8) into Eq. (5), we obtain for the full dual Lagrangian

$$
\begin{aligned}
\mathcal{L}= & -\frac{1}{2 \rho} \sigma_{\tau}^{a} \sigma_{\tau}^{a}-\frac{1}{8 \mu}\left(\sigma_{m}^{a} \sigma_{m}^{a}+\sigma_{m}^{a} \sigma_{a}^{m}-\frac{2 v}{1+v} \sigma_{a}^{a} \sigma_{b}^{b}\right) \\
& +b_{\lambda}^{a} J_{\lambda}^{a}+h_{\lambda} \Theta_{\lambda}+u_{\mathrm{sm}}^{a}\left(\partial_{\mu} \sigma_{\mu}^{a}\right)+\omega_{\mathrm{sm}}\left(\epsilon_{a m} \sigma_{m}^{a}\right) .
\end{aligned}
$$

This is just the standard action for elasticity written in stress fields, having the constraints Eqs. (2) and (3) explicit in terms of the Lagrange multipliers $u_{\mathrm{sm}}^{a}, \omega_{\mathrm{sm}}$. These are resolved by the stress gauge fields where $b_{\lambda}^{a}$ is sourced as usual by the dislocation current, but the notable feature is the term representing the disclination current $\Theta_{\lambda}=\epsilon_{\lambda \mu \nu} \partial_{\mu} \partial_{\nu} \omega_{\text {sing }}$, enumerating the singular part of the rotation field ${ }^{23,25}$ sourcing the torque stress gauge field $h_{\lambda}$. The physical stress fields $\sigma_{\mu}^{a}$ and $\tau_{\mu}$ are invariant under the following gauge transformations involving arbitrary fields $f^{a}$ and $g$ :

$$
\begin{aligned}
b_{\lambda}^{a} & \rightarrow b_{\lambda}^{a}+\partial_{\lambda} f^{a}, \\
h_{m} & \rightarrow h_{m}+\epsilon_{m a} f^{a}, \\
h_{\lambda} & \rightarrow h_{\lambda}+\partial_{\lambda} g .
\end{aligned}
$$


Substituting into Eq. (9), we find the conservation laws for the defect currents, ${ }^{23}$

$$
\begin{gathered}
\partial_{\mu} J_{\mu}^{a}=\epsilon_{m a} \Theta_{m}, \\
\partial_{\mu} \Theta_{\mu}=0 .
\end{gathered}
$$

which appear here as natural consequences of the gauge symmetries Eqs. (10) and (11).

It is an old wisdom that disclinations are infinite-energy defects in solids (the crankshaft effect), as we can now address in gauge theory language. After substituting Eq. (4), Eq. (9) acquires a Maxwell-like form. Going to momentum space where $p=\left(\frac{1}{c_{\mathrm{T}}} \omega, \mathbf{q}\right)$, by exploiting Eq. (6) in combination with Eq. (8) one straightforwardly finds that the part of the Lagrangian involving torque stress gauge fields has the form $\sim \mathbf{q}^{4} h_{\tau}^{\dagger} h_{\tau}+h_{\tau} \Theta_{\tau}$. Thus the torque stress acts over very short distances only, as indicated by

$$
\left\langle h_{\tau}(p) h_{\tau}(0)\right\rangle=\left.\frac{1}{Z} \frac{\delta^{2} Z[\Theta]}{\delta \Theta_{\tau}(0) \delta \Theta_{\tau}(p)}\right|_{\Theta \rightarrow 0}=\frac{\kappa+\mu}{2 \kappa \mu} \frac{1}{q^{4}} .
$$

This goes hand-in-hand with the energy of a static disclinationantidisclination pair increasing quadratically with their separation, which agrees with Ref. 33. This is quite like the confinement phenomenon in compact Yang-Mills theories, except that there the potential is linear. Although the mechanism is in detail rather different, the physical meaning of the confinement phenomenon is very similar in both cases. In QCD the quarks are the sources of color forces mediated by gluons, but the confining state is "color-undeformable," only allowing for color singlets, while quarks and gluons do not have physical existence in the infrared. In the solid, disclinations are like quarks and the torque gauge fields are like gluons, but the vacuum of the solid is a "torque-gauge singlet" and can therefore be used as crankshaft.

\section{QUANTUM $p$-ATIC}

Can the torque gauge field be deconfined? The reader might anticipate the answer: In the superconducting quantum $p$-atic, disclinations should occur as the topological defects, while our torque stress gauge field should dualize into the massless rotational Goldstone mode. We will show this by explicitly mobilizing the Abelian-Higgs duality technology which directly applies to the problem of quantum melting of the bosonic crystal into the $p$-atic superconductor in $(2+1) \mathrm{D}$, extending the $2 \mathrm{D}$ topological melting theory. ${ }^{20-22}$ Dislocations uniquely restore translational symmetry. The $p$ atic fluid can therefore always be considered as a crystal where the dislocations have spontaneously proliferated. In $(2+1) \mathrm{D}$ the dislocations are themselves bosonic point particles and when they proliferate they will Bose-condense. This Bose condensate itself must be a superfluid, supporting zero sound compressional modes. Since the stress gauge fields mediating long-range interactions between the dislocations are like photons, this dislocation condensate is a (relativistic) "dual stress superconductor." Elsewhere we have explored the gross properties of such bosonic quantum liquid crystals. ${ }^{26,30,34}$ In short summary, the shear photons acquire a Higgs mass $\Omega$, indicating that the shear forces in the $p$-atic become short ranged after restoring translational symmetry, while in principle a massive, propagating shear photon should be present. ${ }^{27,28,34}$ Since compressional stress (as opposed to shear stress) does not couple to dislocations, ${ }^{35}$ the longitudinal phonon of the solid turns into a pure zero-sound mode, that coincides with the phase mode of the superfluid. Upon coupling to electromagnetism it can also be shown that the dislocation condensate shows a genuine Meissner effect-it is a regular superconductor. ${ }^{26,28,34}$

Lacking the torque gauge field formalism introduced above, in this older work the fate of the rotations and/or torque in the dislocation condensate was rather obscure. Using torque gauge fields this now becomes explicitly tractable and we find that the mechanism by which the rotational Goldstone is "born" in the $p$-atic state is elegant but counterintuitive: It is formed from the longitudinal modes of the dislocation condensate itself. This is rooted in two special properties of this condensate. First, as in all Abelian-Higgs type dualities, there is only one velocity (the phonon velocity) and such condensates are truly relativistic Higgs phases where the condensate phase mode propagates with the same velocity as the photons. Therefore, the condensate mode is eaten by the gauge field, giving rise to an additional (massive) "longitudinal photon." Second, the dislocations carry Burgers vectors and therefore the condensate has multiple components. The rotational point group symmetry imposes a unique way to construct the dislocation condensate corresponding with the $p$-atic: ${ }^{27-29,36,37}$ the dislocation Burgers vectors have to orient along the directions associated with the crystal point group and the $p$-atic condensate is formed by populating all these directions equally. (It is also possible to take a different condensate superposition in dislocation Hilbert space, which addresses other, less isotropic $p$-atics. ${ }^{37}$ ) Taking these requirements it follows from symmetry reasoning that the Josephson form of the Higgs term of the $p$-atic condensate has to have the form

$$
\mathcal{L}_{\text {Higgs }}=-\frac{1}{2 \mu} \Omega^{2}\left(\partial_{\lambda} \phi^{a}-b_{\lambda}^{a}\right)^{2} .
$$

The dislocation condensate fields are $\Phi^{a}=\left|\Phi^{a}\right| e^{i \phi_{a}}$, where $a$ indicates the Burgers directions, $\Omega \sim\left|\Phi^{a}\right|$ is the Higgs mass, and $\phi^{a}$ is the phase variable of the condensate field. The complete action for the quantum $p$-atic is obtained by adding Eq. (9) and omitting the dislocation source term. Focusing on the symmetric gauge and condensate fields $\left(\mathcal{L}_{\text {symmetric }}\right)$ one discovers the physics discussed in the previous section. However, by employing torque stress fields it becomes easy to address the rotational sector as well. The notable feature is that the Higgs term Eq. (15), which usually encodes for mass, is now the origin of the massless rotational Goldstone mode.

The proof is easy. By taking the unitary gauge fix $b_{\lambda}^{a} \rightarrow$ $b_{\lambda}^{a}+\partial_{\lambda} \phi^{a}$ the condensate modes are shuffled into the gauge sector, leaving

$$
\mathcal{L}_{\text {Higgs }}=-\frac{1}{2 \mu} \Omega^{2}\left(b_{\lambda}^{a}\right)^{2} .
$$

Clearly, all components of the stress gauge field obtain a mass $\frac{\Omega^{2}}{\mu}$. From the definition (6) we have the relations,

$$
\tau_{\tau}=-b_{x}^{x}-b_{y}^{y}, \quad \tau_{x}=b_{\tau}^{x}, \quad \tau_{x}=b_{\tau}^{y} .
$$


Substituting Eq. (17) into Eq. (16), we find

$$
\begin{aligned}
\mathcal{L}_{\text {Higgs }}= & \frac{1}{2} \frac{\Omega^{2}}{\mu}\left[\frac{1}{2} \tau_{\tau}^{2}+\tau_{x}^{2}+\tau_{y}^{2}+\frac{1}{2}\left(b_{x}^{y}-b_{y}^{x}\right)^{2}\right. \\
& \left.+\frac{1}{2}\left(b_{x}^{x}-b_{y}^{y}\right)^{2}+\frac{1}{2}\left(b_{x}^{y}+b_{y}^{x}\right)^{2}\right] .
\end{aligned}
$$

Here $b_{x}^{y}-b_{y}^{x}$ corresponds to compression stress, which remains massless since it does not couple to dislocations at all. This can be handled by imposing the so-called glide contraint. ${ }^{35}$ The components $b_{x}^{x}-b_{y}^{y}$ and $b_{x}^{y}+b_{y}^{x}$ represent the gapping out of shear stress, the hallmark of the demise of the crystal. More importantly, an emergent dynamical term appears as

$$
\mathcal{L}_{R}=-\frac{1}{2} \frac{\Omega^{2}}{4 \mu} \tau_{\mu}^{2}
$$

Note that the temporal component in Eq. (18) has an additional factor $\frac{1}{2}$, which indicates that we should rescale the velocity from $c_{T}$ to $c_{R}=c_{T} / \sqrt{2}$, which we have done in going to the above equation. The reason for this factor is that there is that both spatial directions contribute to $\tau_{\tau}$ in Eq. (17) in this isotropic condensate; in three dimensions, we expect a factor of $1 / \sqrt{3}$ in the velocity for the rotational Goldstone modes. Applying Eq. (8) and including the disclination current from Eq. (9), we find that the long-wavelength rotational dynamics is just governed by the Higgs term Eq. (16) acquiring the simple form

$$
\mathcal{L}_{\text {Rotational }}=-\frac{1}{2} \frac{\Omega^{2}}{4 \mu}\left(\tilde{F}_{\mu \nu} \tilde{F}_{\mu \nu}\right)+h_{\mu} \Theta_{\mu} .
$$

where $\tilde{F}_{\mu \nu}=\partial_{\mu} h_{v}-\partial_{\nu} h_{\mu}$ is parameterizing the torque stress term $\tau_{\mu} \tau_{\mu}$ in terms of the torque stress gauge fields $h_{\mu}$ being sourced by the disclination currents $\Theta_{\mu}$. The form of Eq. (20), besides periodicity issues that are kept implicit here, indicates that disclinations in $(2+1) \mathrm{D}$ are just like vortices, and the rotational Goldstone mode is just like the phase mode in the superfluid, giving rise to the usual logarithmic interactions. The long-range orientational order finally takes the form of a truly propagating Goldstone mode. However, the origin of the rotational rigidity is remarkable. Performing the mathematical duality operation backwards to the "strain side," the elastic action associated with the rotational rigidity becomes that of a free massless scalar field $\phi$,

$$
\mathcal{L}_{p \text {-atic }}=\frac{1}{2} \frac{2 \mu}{\Omega^{2}}\left[\left(\frac{1}{c_{\mathrm{R}}} \partial_{\tau} \phi\right)^{2}+\left(\partial_{m} \phi\right)^{2}\right] .
$$

The conclusion is that besides the shear modulus $\mu$ and the mass density $\rho$ of the background crystal (recall that $c_{\mathrm{R}}^{2}=$ $c_{\mathrm{T}}^{2} / 2=\mu / 2 \rho$ ) this just depends on the Higgs mass $\Omega$ of the dislocation condensate. The rotational modulus $K=2 \mu / \Omega^{2}$ is diverging upon approaching the quantum phase transition to the crystal, which is perfectly reasonable given that the crystal is completely undeformable in this regard.

\section{DISCUSSION}

In the seemingly mundane context of elasticity of crystals and liquid crystals we have uncovered a remarkably rich and elegant gauge field-theoretical structure, explaining the rotational rigidity of the superconducting liquid crystal in terms of a deconfinement transition from the confining solid. Recently, another work has appeared that gives a systematic categorization of absent Goldstone modes when broken symmetry generators are not independent, ${ }^{38}$ consistent with the confinement of rotational modes in the crystal.

Comparing with earlier works in 3D classical elasticity via Wick rotation, several notable features arise. First, in earlier works rotational degrees of freedom are introduced phenomenologically, whereas we derive them through the mathematically precise dual Ginzburg-Landau phase transition. The velocity $c_{\mathrm{R}}$ in Eq. (21) is unique in this isotropic choice of condensate, whereas in Ref. 31 the Wick-rotated velocity was an arbitrary ratio of the core energies between screw and edge dislocations. In the 2D quantum $p$-atic, screw dislocations are absent since singularities in the temporal direction are forbidden. Second, as the quantum $p$-atic is a stress superconductor at the same time, hydrodynamic sound is gapped and the rotational Goldstone mode is not overdamped. Third, previously the shear correlator $\langle\omega, \omega\rangle$ is used to interrogate the rotational sector, but it also excites transverse phonons, e.g., Eq. (3.23) in Ref. 31. The torque gauge fields are much cleaner in this respect. The most striking observation is that the dislocation condensate, and not the crystal, supports rotational modes. As the Higgs mass vanishes towards the quantum phase transition, the rotational modes are lost.

How do we test our quantitative prediction Eq. (21) experimentally? The available superconducting nematics ${ }^{13,14,17}$ may be of the strongly correlated kind, but the presence of an ionic lattice breaking the space rotations explicitly is an obscuring factor. This "lattice pinning" of the (uniaxial) director will cause an anisotropy gap in the spectrum of the rotational mode, which has to be small compared to the other scales in order for the continuum theory to be of relevance. A more fundamental complication is that one can only probe the electron nematics through their electromagnetic response. The massive shear mode is observable in linear response, ${ }^{28}$ albeit in a kinematical regime which is beyond the reach of present spectroscopic techniques. Similarly, it is straightforward to compute ${ }^{26,28}$ the coupling of external electromagnetic fields to the rotational mode: it is in principle visible in the transverse dielectric function with the complication that $h_{\lambda}$ only couples to the gradient of the magnetic field $B$ as $\epsilon_{i j} h_{i} \partial_{j} B$. This gradient coupling greatly complicates the direct measurement of this dynamical mode and we leave it as a challenge for the community to find out how to create and interrogate the "platonic" $p$-atic superconductors of the weak-strong duality in the laboratory.

\section{ACKNOWLEDGMENTS}

This work was supported by the Netherlands foundation for Fundamental Research of Matter (FOM) and the Nederlandse Organisatie voor Wetenschappelijk Onderzoek (NWO) via a Spinoza grant. A.J.B. is supported by the Foreign Postdoctoral Researcher program at RIKEN. 
*wu@lorentz.leidenuniv.nl

${ }^{1}$ Y. Nambu, Phys. Rev. 117, 648 (1960).

${ }^{2}$ J. Goldstone, Nuovo Cimento 19, 154 (1961).

${ }^{3}$ T. Brauner, Symmetry 2, 609 (2010).

${ }^{4}$ I. Low and A. V. Manohar, Phys. Rev. Lett. 88, 101602 (2002).

${ }^{5}$ J. Sethna, Statistical Mechanics: Entropy, Order Parameters and Complexity, Oxford Master Series in Statistical, Computational, and Theoretical Physics (Oxford University Press, Oxford, 2006).

${ }^{6}$ B. G. Chen, G. P. Alexander, and R. D. Kamien, Proc. Natl. Acad. Sci. 106, 15577 (2009).

${ }^{7}$ P. de Gennes and J. Prost, The Physics of Liquid Crystals, International Series of Monographs on Physics (Clarendon, Oxford, 1995).

${ }^{8} \mathrm{P}$. Chaikin and T. Lubensky, Principles of Condensed Matter Physics (Cambridge University Press, Cambridge, 2000).

${ }^{9}$ J.-M. Park and T. C. Lubensky, Phys. Rev. E 53, 2648 (1996).

${ }^{10}$ D. Forster, Hydrodynamic Fluctuations, Broken Symmetry, and Correlation Functions (W. A. Benjamin, Reading, MA, 1975).

${ }^{11}$ S. Kivelson, E. Fradkin, and V. Emery, Nature (London) 393, 550 (1998).

${ }^{12}$ E. Fradkin, S. A. Kivelson, M. J. Lawler, J. P. Eisenstein, and A. P. Mackenzie, Annu. Rev. Cond. Mat. Phys. 1, 153 (2010).

${ }^{13}$ Y. Ando, K. Segawa, S. Komiya, and A. N. Lavrov, Phys. Rev. Lett. 88, 137005 (2002).

${ }^{14}$ V. Hinkov, D. Haug, B. Fauqué, P. Bourges, Y. Sidis, A. Ivanov, C. Bernhard, C. T. Lin, and B. Keimer, Science 319, 597 (2008).

${ }^{15}$ M. J. Lawler, K. Fujita, J. Lee, A. R. Schmidt, Y. Kohsaka, C. K. Kim, H. Eisaki, S. Uchida, J. C. Davis, J. P. Sethna et al., Nature (London) 466, 347 (2010).

${ }^{16}$ A. Mesaros, K. Fujita, H. Eisaki, S. Uchida, J. C. Davis, S. Sachdev, J. Zaanen, M. J. Lawler, and E.-A. Kim, Science 333, 426 (2011).
${ }^{17}$ T.-M. Chuang, M. P. Allan, J. Lee, Y. Xie, N. Ni, S. L. Bud'ko, G. S. Boebinger, P. C. Canfield, and J. C. Davis, Science 327, 181 (2010).

${ }^{18}$ J.-H. Chu, H.-H. Kuo, J. G. Analytis, and I. R. Fisher, Science 337, 710 (2012).

${ }^{19}$ V. Oganesyan, S. A. Kivelson, and E. Fradkin, Phys. Rev. B 64, 195109 (2001).

${ }^{20}$ B. I. Halperin and D. R. Nelson, Phys. Rev. Lett. 41, 121 (1978).

${ }^{21}$ D. R. Nelson and B. I. Halperin, Phys. Rev. B 19, 2457 (1979).

${ }^{22}$ A. P. Young, Phys. Rev. B 19, 1855 (1979).

${ }^{23}$ H. Kleinert, Gauge Fields in Condensed Matter, Vol. II Stress and Defects (World Scientific, Singapore, 1989).

${ }^{24}$ H. Kleinert and J. Zaanen, Phys. Lett. A 324, 361 (2004).

${ }^{25} \mathrm{H}$. Kleinert, Mulivalued Fields in Condensed Matter, Electromagnetism, and Gravitation (World Scientific, Singapore, 2008).

${ }^{26}$ J. Zaanen, Z. Nussinov, and S. Mukhin, Ann. Phys. (NY) 310, 181 (2004).

${ }^{27}$ V. Cvetkovic and J. Zaanen, Phys. Rev. Lett. 97, 045701 (2006).

${ }^{28}$ V. Cvetkovic, Z. Nussinov, S. Mukhin, and J. Zaanen, Europhys. Lett. 81, 27001 (2008).

${ }^{29}$ V. Cvetkovic, Ph.D. thesis, Leiden University, 2006 (unpublished), http://hdl.handle.net/1887/4456.

${ }^{30}$ J. Zaanen and A. Beekman, Ann. Phys. (NY) 327, 1146 (2012).

${ }^{31}$ M. C. Marchetti and D. R. Nelson, Phys. Rev. B 41, 1910 (1990).

${ }^{32}$ M. C. Marchetti and L. Radzihovsky, Phys. Rev. B 59, 12001 (1999).

${ }^{33}$ H. S. Seung and D. R. Nelson, Phys. Rev. A 38, 1005 (1988).

${ }^{34}$ V. Cvetkovic and J. Zaanen, Phys. Rev. B 74, 134504 (2006).

${ }^{35}$ V. Cvetkovic, Z. Nussinov, and J. Zaanen, Philos. Mag. 86, 2995 (2006).

${ }^{36}$ V. Cvetkovic, A. Beekman, K. Wu, and J. Zaanen (unpublished).

${ }^{37}$ C. Mathy and F. Bais, Ann. Phys. (NY) 322, 709 (2007).

${ }^{38}$ H. Watanabe and H. Murayama, Phys. Rev. Lett. 110, 181601 (2013). 